\title{
Manow, Philip: (Ent-)Demokratisierung der Demokratie, 215 S., Suhrkamp, Berlin 2020.
}

\author{
Dirk Jörke \\ Angenommen: 17. Dezember 2020 / Online publiziert: 5. Januar 2021 \\ (C) Der/die Autor(en) 2021
}

Der Bremer Politikwissenschaftler Philip Manow hat auf dem Höhepunkt der Populismusdiskussion im Jahr 2018 ein viel beachtetes Buch vorgelegt, in dem er eine politökonomische Erklärung des Populismus entwickelt hat. Seine Kernthese ist, dass sowohl makrogesellschaftliche Strukturen wie Wohlfahrtssysteme, als auch individuelle Erfahrungen wie erlittene Arbeitslosigkeit für die Wahl von populistischen Parteien ursächlich sind. Er hat damit stärker kulturellen Erklärungen widersprochen. Auch in seinem neuen Buch mit dem Titel ,(Ent-)Demokratisierung der Demokratie“ zeigt Manow sich als streitbarer und provokanter Analytiker der Gegenwart.

Der Band enthält vier Essays, die teilweise bereits zuvor veröffentlicht worden sind; es handelt sich mithin nicht um ein Buch aus einem Guss. Vielmehr können die einzelnen Kapitel und die Einleitung als Schlaglichter betrachtet werden, die eine Vielzahl von klugen und zugleich provozierenden Gedanken enthalten. Im Einzelnen geht es zunächst um liberale Abwehr- und Abwertungsstrategien gegenüber den nach politischer Beteiligung und sozialer Gerechtigkeit strebenden Arbeitern im 19. Jahrhundert. Manow erinnert eindrucksvoll daran, dass die sogenannte repräsentative Demokratie in Abwehr gegen demokratische Bestrebungen entstanden ist, dass es demnach bei der Erfindung repräsentativer Institutionen vornehmlich darum ging, den „Pöbel“ (S. 36) zunächst von der politischen Teilnahme auszuschließen und ihn auch später - nach den Wahlrechtsausweitungen - durch Filterungsprozesse weiterhin außen vor zu lassen: ,[...] es ist der Trick der Repräsentation, etwas in der Demokratie beständig Anwesendes abwesend zu halten“ (S. 47).

Allein, dieser Mechanismus funktioniere nicht mehr. Warum das so sei, wird im folgenden Kapitel erläutert. Dort schildert Manow unter dem Schlagwort „Das Ende der repräsentativen Politik“ Prozesse der Umgehung repräsentativer Institutionen, also jener Institutionen, die den Willen des „Pöbels“ verfeinern sollten. Anhand einiger illustrativer Beispiele aus der Vergangenheit, wie derjenigen von Jeremy Corbyn, Donald Trump und Emmanuel Macron, fokussiert Manow dabei vor allem auf die zunehmende Etablierung von direkten Wahlen politischer Führungspersonen. Die These ist hier folglich, dass es zu einer zunehmenden Entmachtung von Par-

D. Jörke $(\bowtie)$

Technische Universität Darmstadt, Darmstadt, Deutschland

E-Mail: joerke@pg.tu-darmstadt.de 
teiapparaten gekommen sei; ein Prozess, der sich zwar als „Demokratisierung der Demokratie“ (S. 28) beschreiben lässt, der aber eben auch zu einem „Kontrollverlust“ (S. 110) und in der Konsequenz zur titelgebenden ,,(Ent-)Demokratisierung der Demokratie" geführt habe. Wer nun vermutet, hier schreibt ein liberaler Nostalgiker und Moralist, der das Versagen wichtiger repräsentativer Institutionen beklagt, sieht sich in den beiden abschließenden Kapiteln freilich eines Besseren belehrt.

Denn in diesen Kapiteln beklagt Manow, wenngleich nicht durchgehend und zumindest für den Rezensenten bisweilen auch in etwas widersprüchlicher Form, die zunehmende Moralisierung des politischen Streits in der Demokratie. Zunächst gewinnt man nämlich bei der Lektüre den Eindruck, hier würden nur altbekannte Muster der Populismusdiskussion reproduziert, zum Beispiel, dass die politische Macht in der Demokratie ,ein grundsätzlich leerer Ort“ (S. 122) zu sein habe, die Anprangerung des Regierungsstils von Trump oder der vermeintliche Putsch, mit dem Boris Johnson das widerstrebende britische Unterhaus ausgetrickst habe. Doch schon bald verlässt Manow diese ausgetretenen Pfade und kommt zu Beobachtungen wie derjenigen, dass es unsere ,demokratische Pflicht“" sei, die Gesellschaft in zwei Gruppen zu teilen, nämlich in diejenigen, ,die die Gesellschaft in zwei Gruppen teilen [gemeint sind die Populisten mit ihrer Leitunterscheidung zwischen ,gutem“ Volk und ,bösen“ Eliten, D.J.] und in die, die das nicht tun“ (S. 143). Man spürt bei der Lektüre die Freude, die der Verfasser bei der Formulierung dieser und zahlreicher ähnlicher Passagen gehabt haben muss. Kurzum, Manow hält dem Mainstream der wissenschaftlichen wie öffentlichen Debatte einen Spiegel vor, in dem die ,aufrichtigen' Demokraten gar nicht so anders aussehen, als jene, von denen sie sich mit den allzu bekannten Floskeln abzugrenzen versuchen. Diesen Aspekt greift er in der Schlussbetrachtung wieder auf, in der er völlig zu Recht das institutionentheoretische Defizit der sogenannten radikalen Demokratietheorie kritisiert. Auch diese flüchte sich in „Pathosformeln“ (S. 172), die es Autoren wie Lesern gestatte, sich auf der ,richtigen“ moralischen Seite zu verorten. Die ,radikale Demokratie“ ist daher mehr Teil des Problems als Teil der Lösung.

Auch wenn die dahinterstehenden Einsichten nicht gänzlich neu sind, ist das erfrischend zu lesen und kann nicht oft genug betont werden. Beides gilt schließlich ebenso für den Hinweis auf die entdemokratisierenden Konsequenzen des europäischen Supranationalismus (S. 162). Obwohl Manow diesen Aspekt mehr andeutet als systematisch darlegt, so lässt sich die aktuelle populistische Revolte und das Implodieren der filternden Institutionen der repräsentativen Demokratie eben auch darauf zurückführen, dass die Erzählung vom souveränen Staatsvolk im europäischen Mehrebenensystem mehr als unplausibel geworden ist. Und auch allen mit großem demokratietheoretischen Aufwand betriebenen Bemühungen, die Europäische Union ,zu demokratisieren“, zum Trotz, wird sich daran absehbar wohl wenig ändern. Insofern ist es nur konsequent, wenn Manow gegen Ende seines Essays darauf verweist, dass Demokratie eines nationalstaatlichen Rahmens bedarf. Doch, so führt er weiter aus, diese „Grundbedingung moderner, repräsentativer Demokratie wird allerdings selten betont und findet neuerdings nur noch als Skandal Erwähnung“ (S. 159).

Funding Open Access funding enabled and organized by Projekt DEAL. 
Open Access Dieser Artikel wird unter der Creative Commons Namensnennung 4.0 International Lizenz veröffentlicht, welche die Nutzung, Vervielfältigung, Bearbeitung, Verbreitung und Wiedergabe in jeglichem Medium und Format erlaubt, sofern Sie den/die ursprünglichen Autor(en) und die Quelle ordnungsgemäß nennen, einen Link zur Creative Commons Lizenz beifügen und angeben, ob Änderungen vorgenommen wurden.

Die in diesem Artikel enthaltenen Bilder und sonstiges Drittmaterial unterliegen ebenfalls der genannten Creative Commons Lizenz, sofern sich aus der Abbildungslegende nichts anderes ergibt. Sofern das betreffende Material nicht unter der genannten Creative Commons Lizenz steht und die betreffende Handlung nicht nach gesetzlichen Vorschriften erlaubt ist, ist für die oben aufgeführten Weiterverwendungen des Materials die Einwilligung des jeweiligen Rechteinhabers einzuholen.

Weitere Details zur Lizenz entnehmen Sie bitte der Lizenzinformation auf http://creativecommons.org/ licenses/by/4.0/deed.de.

\section{Nassehi, Armin: Muster. Theorie der digitalen Gesellschaft, 352 S., Beck, München 2019.}

\section{Martin Endreß}

Angenommen: 5. Januar 2021 / Online publiziert: 21. Januar 2021

(C) Gesellschaft zur wissenschaftlichen Förderung politischer Literatur e.V. and the Author(s) 2021

Soziologische Zeit- oder Gegenwartsdiagnosen sind ebenso en vogue wie umstritten. Entsprechend ist man geneigt, einem soziologischen Autor Kredit einzuräumen, wenn er dieses schwierige Verhältnis der Disziplin zu einem ihrer Genres reflexiv aufnimmt. Zumindest im Prinzip, denn eine latente Entwertung erfährt diese Selbstbeobachtung durch die selbstgewisse Rahmung von Armin Nassehi, dass er „vermutlich skeptisch“ auf ein Buch zur „Theorie der digitalen Gesellschaft“ reagiert hätte, wenn er ,es nicht selbst geschrieben hätte“ (S. 11). An Selbstbewusstsein mangelt es nicht und die kommunizierten Ansprüche verhalten sich komplementär dazu: keine ,Studie über ... oder ,Untersuchung zu ...', sondern eine „Theorie“, und zudem erneut eine „Gesellschaftsdiagnose“, die diese ,an einem Merkmal festzumachen" verspricht (ebd.).

Doch jenseits vorschneller gegenwartsanalytischer Zuspitzungen ist Nassehis Anliegen ein weiterreichendes: „Ich werde behaupten, dass die gesellschaftliche Moderne immer schon digital war, dass die Digitaltechnik also letztlich nur die logische

\footnotetext{
M. Endreß $(\bowtie)$

Universität Trier, Trier, Deutschland

E-Mail: endress@uni-trier.de
} 